\title{
Brane $f(\mathcal{R})$ gravity
}

\author{
Ahmad Borzou*, Hamid Reza Sepangi ${ }^{\dagger}$, Shahab Shahidi ${ }^{\ddagger}$ and Razieh Yousefi ${ }^{\S}$ \\ Department of Physics, Shahid Beheshti University, Evin, Tehran 19839, Iran
}

October 31, 2018

\begin{abstract}
We consider a brane world scenario in which the bulk action is assumed to have the form of a generic function of the Ricci scalar $f(\mathcal{R})$ and derive the resulting Einstein field equations on the brane. In a constant curvature bulk a conserved geometric quantity appears in the field equations which can be associated with matter. We present cosmological and spherically symmetric solutions by assuming specific forms for $f(\mathcal{R})$ and show that the former can explain an accelerated expanding universe while the latter may account for galaxy rotation curves.
\end{abstract}

\section{Introduction}

The roots of the idea that our four-dimensional universe might be a three-brane embedded in a higher dimensional bulk can be traced back to string theory [1]. In fact, the present day brane world scenarios in their various flavors are influenced, in one way or other, by string theory. One of the most successful of such higher dimensional models is that proposed by Randall and Sundrum where our 4-dimensional world is considered as a brane in a 5-dimensional bulk having the geometry of an AdS space 2, 3. They were successful in explaining what is known as the hierarchy problem; the enormous disparity between the strength of the fundamental forces. The Randall-Sundrum (RS) scenario has had a great impact on our understanding of the universe and has brought higher dimensional gravitational theories to the fore. In the RS type models, only gravitons can wander into the bulk whereas ordinary matter and gauge fields are confined to the brane. Such a confinement is achieved by the application of the Israel junction conditions. Later on, Shiromizu, Maeda and Sasaki (SMS) [5] showed how to systematically project the Einstein field equations, written in the bulk, onto the brane using the Gauss-Codazzi equations and the Israel junction conditions [4, assuming $\mathbb{Z}_{2}$ symmetry. These field equations differ from the standard Einstein field equations in $4 D$ in that they have additional terms like $\pi_{\mu \nu}$ which depend on the energy-momentum tensor on the brane and the electric part of the Weyl tensor $E_{\mu \nu}$, leading to the appearance of a quadratic term in the friedmann equation 6]. Brane world scenarios have paved the way for a new understanding of dark matter among other things for which static, spherically symmetric solutions of the field equations on the brane are of primary interest [7. As an example, the galaxy rotation curves have been accounted for in 8 by studying the vacuum solutions in a brane world scenario in which the brane admits a family of conformal Killing vector fields, leading to exact solutions of the field equations in parametric forms. These solutions were then used to explain the galaxy rotation curves without postulating the existence of dark matter.

Other brane world scenarios have emerged since the seminal work of RS, the most influential of which is the model proposed by Dvali-Gabadadze-Porrati (DGP) 9]. In this model the bulk space is assumed to be flat but there is an additional $4 D$ induced gravity term appearing in the action. The cosmological implications of the DGP model was first studied in [10 where it was shown that the universe experiences a self accelerating phase at late times. The DGP model predicts modifications to

\footnotetext{
*Electronic address: a.borzou@mail.sbu.ac.ir

$\dagger$ Electronic address: hr-sepangi@sbu.ac.ir

$\ddagger$ Electronic address: sh.shahidi@mail.sbu.ac.ir

$\S$ Electronic address: raz.yousefi@mail.sbu.ac.ir
} 
gravity at large distances, in contrast to RS type models which modify gravity at small scales. Other brane world models have been proposed in which the condition of $\mathbb{Z}_{2}$ symmetry is relaxed and the confinement of matter to the brane is achieved through the use of a confining potential [11, 12, 13. Brane world models in the context of higher order gravity have also been studied [14, 15, 16] where the Gauss-Bonnet term is included in the bulk action and the $4 D$ field equations are derived in the usual manner.

Theories of gravity in which the Einstein-Hilbert $(\mathrm{EH})$ action is replaced with a generic function of $\mathcal{R}$ have been gaining momentum in the last few years. In this regard, brane world scenarios have also been proposed both in the context of $5 D$ scalar-tensor type models [17] where such a function of the Ricci scalar is assumed to form the action in the bulk and DGP models 19 where the induced action on the brane contains the $f(\mathcal{R})$ term. However, the study of a RS type model with $f(\mathcal{R})$ as the action in bulk space has been lacking. Such a setup would be of interest since there is no a priori reason as to why the action in $5 \mathrm{D}$ should be the standard $\mathrm{EH}$, in contrast to $4 \mathrm{D}$ where the standard $\mathrm{EH}$ action seems to be, to a large extent, adequate in describing the cosmos. It is therefore the purpose of the present work to obtain the Einstein field equations on the brane from a $f(\mathcal{R})$ action in the bulk within the framework of the RS model, using the SMS procedure. In doing so, we obtain the term $Q_{\mu \nu}$ which completely depends on $f(\mathcal{R})$ and its derivatives. Interestingly, for a conformally flat bulk space, this quantity is conserved, that is, $\nabla^{\mu} Q_{\mu \nu}=0$. This is of course welcome since one may identify such a term with some kind of matter whose origins lie in the geometry of the bulk. It should also be mentioned that the work presented in [18] is an attempt to assume a generic $f(\mathcal{R})$ function as the action for the bulk space in the presence of a real scalar field. However, their method of obtaining the field equations on the brane is based on assuming a specific metric for the bulk which makes dealing with the problem different from what we propose to do.

In what follows, we first derive the field equations on the brane from a generic $f(\mathcal{R})$ action assumed for the bulk. We then study both the static, spherically symmetric and cosmological solutions of the resulting field equations. Conclusions are drawn in the last section.

\section{Effective field equations on the brane}

We start by deriving the Einstein field equations on the brane, assuming the following action for the bulk

$$
S=\int \mathrm{d}^{5} x \sqrt{-\mathrm{g}}\left[f(\mathcal{R})+\mathcal{L}_{m}\right]
$$

where $\mathcal{L}_{m}$ is the matter lagrangian, g is the bulk metric and $\mathcal{R}$ is the bulk Ricci scalar. After variation of $S$ with respect to the bulk metric $\mathrm{g}_{A B}$, we obtain

$$
\begin{aligned}
f^{\prime}(\mathcal{R}) \mathcal{R}_{A B} & -\frac{1}{2} \mathrm{~g}_{A B} f(\mathcal{R})+\mathrm{g}_{A B} \square f^{\prime}(\mathcal{R}) \\
& -\nabla_{A} \nabla_{B} f^{\prime}(\mathcal{R})=\kappa_{5}^{2} T_{A B},
\end{aligned}
$$

where a prime represents derivative with respect to the argument. Rearranging the above equation, we obtain the effective Einstein field equations in the bulk

$$
G_{A B} \equiv \mathcal{R}_{A B}-\frac{1}{2} \mathcal{R g}_{A B}=T_{A B}^{t o t}
$$

where

$$
\begin{aligned}
T_{A B}^{t o t} & =\frac{1}{f^{\prime}(\mathcal{R})}\left[\kappa_{5}^{2} T_{A B}-\left(\frac{1}{2} \mathcal{R} f^{\prime}(\mathcal{R})-\frac{1}{2} f(\mathcal{R})\right.\right. \\
& \left.\left.+\square f^{\prime}(\mathcal{R})\right) \mathrm{g}_{A B}+\nabla_{A} \nabla_{B} f^{\prime}(\mathcal{R})\right] .
\end{aligned}
$$

To obtain the field equations on the brane we start by invoking the Gauss equations [20]

$$
R_{\beta \gamma \delta}^{\alpha}=\mathcal{R}_{B C D}^{A} h_{A}^{\alpha} h_{\beta}^{B} h_{\gamma}^{C} h_{\delta}^{D}+K_{\gamma}^{\alpha} K_{\beta \delta}-K_{\delta}^{\alpha} K_{\beta \gamma},
$$


where $h_{A B}=\mathrm{g}_{A B}-n_{A} n_{B}$ is the induced metric on the brane, $n^{A}$ is the unit vector normal to the four-dimensional brane, $R_{\beta \gamma \delta}^{\alpha}$ is the Riemann tensor on the brane and $K_{\mu \nu}=h_{\mu}^{A} h^{B}{ }_{\nu} \nabla_{A} n_{B}$ is the extrinsic curvature. After contracting equation (5) one obtains

$$
\begin{aligned}
R_{\mu \nu} & =\mathcal{R}_{A B} h_{\mu}^{A} h^{B}{ }_{\nu}-\mathcal{R}_{B C D}^{A} n_{A} h_{\mu}^{B} n^{C} h_{\nu}^{D} \\
& +K K_{\mu \nu}-K_{\mu}^{\alpha} K_{\nu \alpha},
\end{aligned}
$$

and

$$
\begin{aligned}
G_{\mu \nu} & =\left[\mathcal{R}_{A B}-\frac{1}{2} \mathrm{~g}_{A B} \mathcal{R}\right] h^{A}{ }_{\mu} h_{\nu}^{B}+\mathcal{R}_{A B} n^{A} n^{B} h_{\mu \nu} \\
& +K K_{\mu \nu}-K_{\mu}^{\rho} K_{\nu \rho}-\frac{1}{2} h_{\mu \nu}\left(K^{2}-K^{\alpha \beta} K_{\alpha \beta}\right) \\
& -\mathcal{R}_{B C D}^{A} n_{A} n^{C} h_{\mu}^{B} h_{\nu}^{D} .
\end{aligned}
$$

Now, let us take the usual decomposition of the Riemann tensor into the Ricci tensor, Ricci scalar and the Weyl tensor $\mathcal{C}_{A B C D}$ as [5]

$$
\begin{aligned}
\mathcal{R}_{A B C D} & =\frac{2}{3}\left(\mathrm{~g}_{A[C} \mathcal{R}_{D] B}-\mathrm{g}_{B[C} \mathcal{R}_{D] A}\right) \\
& -\frac{1}{6} \mathrm{~g}_{A[C} \mathrm{g}_{D] B} \mathcal{R}+\mathcal{C}_{A B C D}
\end{aligned}
$$

and using equation (3) to obtain

$$
\begin{aligned}
& G_{\mu \nu}=\frac{2}{3}\left[T_{A B}^{t o t} h_{\mu}^{A} h_{\nu}^{B}+\left(T_{A B}^{t o t} n^{A} n^{B}-\frac{1}{4} T^{t o t}\right) h_{\mu \nu}\right] \\
& +K K_{\mu \nu}-K_{\mu}^{\sigma} K_{\nu \sigma}-\frac{1}{2} h_{\mu \nu}\left(K^{2}-K^{\alpha \beta} K_{\alpha \beta}\right)-E_{\mu \nu}
\end{aligned}
$$

where $T^{t o t}$ is the trace of $T_{A B}^{t o t}$ and

$$
E_{\mu \nu}=\mathcal{C}_{B C D}^{A} n_{A} n^{C} h_{\mu}^{B} h_{\nu}^{D},
$$

is the electric part of the Weyl tensor. If we assume that the brane is located at $y=0$ we can write the energy-momentum tensor as [5]

$$
T_{A B}=-\Lambda g_{A B}+S_{A B} \delta(y),
$$

where $\Lambda$ is the cosmological constant of the bulk and

$$
S_{\mu \nu}=-\lambda h_{\mu \nu}+\tau_{\mu \nu},
$$

with $\tau_{\mu \nu}$ being the brane energy-momentum tensor and $\lambda$ the associated cosmological constant. With the use of equation (11) we can compute the first three terms in equation (9) as

$$
\begin{aligned}
T_{A B}^{t o t} h_{\mu}^{A} h_{\nu}^{B} & =\left[-\frac{1}{f^{\prime}(\mathcal{R})} \kappa_{5}^{2} \Lambda-\frac{1}{2} \mathcal{R}+\frac{1}{2} \frac{f(\mathcal{R})}{f^{\prime}(\mathcal{R})}-\frac{\square f^{\prime}(\mathcal{R})}{f^{\prime}(\mathcal{R})}\right] h_{\mu \nu} \\
& +\left[\frac{\nabla_{A} \nabla_{B} f^{\prime}(\mathcal{R})}{f^{\prime}(\mathcal{R})}\right] h_{\mu}^{A} h^{B}{ }_{\nu}, \\
T_{A B}^{t o t} n^{A} n^{B} h_{\mu \nu} & =\left[-\frac{1}{f^{\prime}(\mathcal{R})} \kappa_{5}^{2} \Lambda-\frac{1}{2} \mathcal{R}+\frac{1}{2} \frac{f(\mathcal{R})}{f^{\prime}(\mathcal{R})}-\frac{\square f^{\prime}(\mathcal{R})}{f^{\prime}(\mathcal{R})}\right] h_{\mu \nu} \\
& +\left[\frac{\nabla_{A} \nabla_{B} f^{\prime}(\mathcal{R})}{f^{\prime}(\mathcal{R})}\right] n^{A} n^{B} h_{\mu \nu},
\end{aligned}
$$




$$
-\frac{1}{4} T^{t o t} h_{\mu \nu}=\left(\frac{5}{4} \frac{1}{f^{\prime}(\mathcal{R})} \kappa_{5}^{2} \Lambda+\frac{5}{8} \mathcal{R}-\frac{5}{8} \frac{f(\mathcal{R})}{f^{\prime}(\mathcal{R})}+\frac{\square f^{\prime}(\mathcal{R})}{f^{\prime}(\mathcal{R})}\right) h_{\mu \nu} .
$$

To compute the last terms in equation (9) we use the Israel junction conditions [4, 5]

$$
\left[K_{\mu \nu}\right]=-\kappa_{5}^{2}\left(S_{\mu \nu}-\frac{1}{3} h_{\mu \nu} S\right),
$$

where $[X]:=\lim _{y \rightarrow 0^{+}} X-\lim _{y \rightarrow 0^{-}} X$ is the jump of the quantity $X$ across the brane. Assuming $\mathbb{Z}_{2}$ symmetry, we can replace the jump in the extrinsic curvature by the value of the extrinsic curvature at the location of the brane, hence

$$
K_{\mu \nu}^{+}=K_{\mu \nu}^{-}=-\frac{\kappa_{5}^{2}}{2}\left(S_{\mu \nu}-\frac{1}{3} h_{\mu \nu} S\right),
$$

Now, by expressing the extrinsic curvature in terms of $\tau_{\mu \nu}$ and $\lambda$ and using the relation

$$
\mathcal{R}=-\frac{5}{f^{\prime}(\mathcal{R})} \kappa_{5}^{2} \Lambda+\frac{5}{2} \frac{f(\mathcal{R})}{f^{\prime}(\mathcal{R})}-4 \frac{\square f^{\prime}(\mathcal{R})}{f^{\prime}(\mathcal{R})},
$$

obtained by contracting equations(2), we finally obtain the effective Einstein equations on the brane

$$
G_{\mu \nu}=-\Lambda_{4} h_{\mu \nu}+8 \pi G_{N} \tau_{\mu \nu}+\kappa_{5}^{2} \pi_{\mu \nu}+Q_{\mu \nu}-E_{\mu \nu},
$$

where

$$
\begin{gathered}
\Lambda_{4}=\frac{1}{2} \kappa_{5}^{2}\left(\Lambda+\frac{1}{6} \kappa_{5}^{2} \lambda^{2}\right), \\
G_{N}=\frac{\kappa_{5}^{4} \lambda}{48 \pi}, \\
\pi_{\mu \nu}=-\frac{1}{4} \tau_{\mu \alpha} \tau_{\nu}^{\alpha}+\frac{1}{12} \tau \tau_{\mu \nu}+\frac{1}{8} h_{\mu \nu} \tau_{\alpha \beta} \tau^{\alpha \beta}-\frac{1}{24} h_{\mu \nu} \tau^{2}, \\
Q_{\mu \nu}=\left[F(\mathcal{R}) h_{\mu \nu}+\frac{2}{3} \frac{\nabla_{A} \nabla_{B} f^{\prime}(\mathcal{R})}{f^{\prime}(\mathcal{R})}\left(h_{\mu}^{A} h^{B}{ }_{\nu}+n^{A} n^{B} h_{\mu \nu}\right)\right]_{y=0},
\end{gathered}
$$

and

$$
\begin{aligned}
F(\mathcal{R}) \equiv & -\frac{4}{15} \frac{\square f^{\prime}(\mathcal{R})}{f^{\prime}(\mathcal{R})}-\frac{1}{10} \mathcal{R}\left(\frac{3}{2}+f^{\prime}(\mathcal{R})\right) \\
& +\frac{1}{4} f(\mathcal{R})-\frac{2}{5} \square f^{\prime}(\mathcal{R}) .
\end{aligned}
$$

We note that for $f(\mathcal{R})=\mathcal{R}$, we obtain the usual induced equations first obtained in [5. We also note that in the case of constant curvature bulk we have $E_{\mu \nu}=0$ and by equation (23) and (24) we obtain

$$
\nabla^{\mu} Q_{\mu \nu}=0,
$$

since $f(\mathcal{R})$ is a constant. This means that we can identify $Q_{\mu \nu}$ as representing the energy-momentum tensor of an unknown type of matter. In the case of an arbitrary curvature bulk we have the following conservation equation

$$
\nabla^{\mu}\left(Q_{\mu \nu}-E_{\mu \nu}\right)=0
$$




\section{$3 \quad f(\mathcal{R})=\mathcal{R}^{n}$ solutions}

In this section we consider static, spherically symmetric and cosmological solutions to the Einstein field equations given by equation (19). In what follows, we start by studying the black hole solutions and then move on to study the cosmological solutions. In all the solutions presented below, we assume a specific form for the function $f(\mathcal{R})$, namely $f(\mathcal{R})=\mathcal{R}^{n}$.

\subsection{Spherically symmetric solutions}

We begin by taking the following static, spherically symmetric bulk metric

$$
d s^{2}=-A(r) d t^{2}+\frac{1}{A(r)} d r^{2}+r^{2} d \theta^{2}+r^{2} \sin \theta^{2} d \phi^{2}+d y^{2} .
$$

If we assume that the brane is devoid of matter then the second and third terms in (19) become zero. Taking the normal vector to the brane as $n^{A}=(0,0,0,0,1)$, the Einstein field equation becomes

$$
G_{\mu \nu}=-\Lambda_{4} h_{\mu \nu}+Q_{\mu \nu}-E_{\mu \nu}
$$

where $Q_{\mu \nu}$ is given by equation (23). Let us take the constant curvature solutions of equation (28) as

$$
A(r)=1+\frac{a}{r}+b r^{2}
$$

The components of $Q_{\nu}^{\mu}$ are then given by

$$
Q_{0}^{0}=Q_{1}^{1}=Q_{2}^{2}=Q_{3}^{3}=\frac{9 b}{5}-\frac{n(-12 b)^{n}}{10}+\frac{(-12 b)^{n}}{4},
$$

where $b$ is a constant depending on the cosmological constant $\Lambda_{4}$ and $n$ and obtained from the equation

$$
\begin{aligned}
& \left(\frac{n}{10}-\frac{1}{4}\right)(-12 b)^{n}+\frac{6 b}{5}+\Lambda_{4}=0, \quad n \neq 1, \\
& b=-\frac{\Lambda_{4}}{3}, \quad n=1 .
\end{aligned}
$$

Equation (29) represents a Schwarzschild-Anti de-Sitter like solution which is compatible with [21], so we can identify $a$ by $-2 M$ as in ordinary schwarzschild solutions. In the case $n=1$ we recover the standard Schwarzschild-Anti de-Sitter solution. In the case of a vanishing brane cosmological constant, we also have the above solution reduced to

$$
b=-\frac{1}{12}\left(\frac{2}{2 n-5}\right)^{\frac{1}{n-1}}, \quad n \neq 1,
$$

depending only on $n$. Therefore, $Q_{\mu \nu}$ plays the role of the cosmological constant in this case. The form of the above solution suggests that, in very general terms, it may be used to explain the galaxy rotation curves.

For the sake of completeness, let us also consider the case where $f(\mathcal{R})=\mathcal{R}+\mathcal{R}^{n}$ which has been considered in many works. In this case, the equation for $B(n)$ is similar to equation (31) except that the coefficient $b$ is replaced by 3 . In the case of vanishing cosmological constant we find

$$
b=-\frac{1}{12}\left(\frac{5}{2 n-5}\right)^{\frac{1}{n-1}}, \quad n \neq 1 .
$$

\subsection{Cosmological solutions}

In order to investigate the cosmological solutions we consider the standard Robertson-Walker metric

$$
d s^{2}=-d t^{2}+a(t)^{2}\left[d r^{2}+r^{2} d \theta^{2}+r^{2} \sin ^{2} \theta d \phi^{2}\right]+d y^{2},
$$




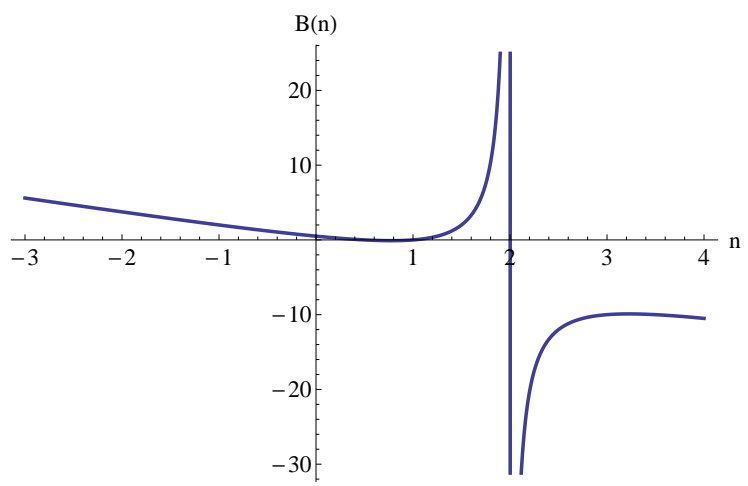

Figure 1: plot of $B(n)$ as a function of $n$.

where $a(t)$ is the scale factor. We now consider a perfect fluid form for the brane matter with a linear equation of state

$$
\begin{aligned}
& \tau_{\nu}^{\mu}=\operatorname{diag}(-\rho(t), p(t), p(t), p(t)), \\
& p(t)=\omega \rho(t),
\end{aligned}
$$

where $\omega$ is a constant. Again, with $n^{A}=(0,0,0,0,1)$ and the assumption that the cosmological constants in both the bulk and brane are zero, we obtain the Einstein field equations as follows

$$
G_{\mu \nu}=\kappa_{5}^{2} \pi_{\mu \nu}+Q_{\mu \nu}-E_{\mu \nu} .
$$

Let us consider a power law form for the scale factor

$$
a(t)=\left(\frac{t}{t_{0}}\right)^{B} .
$$

The Einstein equations then become

$$
\begin{aligned}
& A_{1}+c\left(\frac{6 B(2 B-1)}{t^{2}}\right)^{n} t^{2}=5 \kappa_{5}^{2} a_{1} t^{2} \rho(t)^{2}, \\
& A_{2}+c\left(\frac{6 B(2 B-1)}{t^{2}}\right)^{n} t^{2}=5 \kappa_{5}^{2} a_{2} t^{2} \rho(t)^{2},
\end{aligned}
$$

where

$$
\begin{aligned}
& A_{1}=24 B(2 B-1)(3 B+2 n-1)(B-2 n+2), \\
& A_{2}=8 B(2 B-1)\left(9 B^{2}-5 B-2 n B-12 n+8 n^{2}+4\right), \\
& c=(30-12 n) B^{2}+8 n\left(1-3 n+2 n^{2}\right)-3 B\left(8 n^{2}-10 n+5\right), \\
& a_{1}=B(2 B-1), \\
& a_{2}=-B(2 B-1)(2 \omega+1) .
\end{aligned}
$$

The solution is given by

$$
\omega=-1 \quad \text { for } B \neq 0, \quad B(n)=\frac{1-3 n+2 n^{2}}{2-n} .
$$

In figure 1 we have plotted $B(n)$ as a function of $n$. For an accelerating universe we must have $B>1$. Now, using equation (40) we obtain

$$
\rho(t)^{2}=\frac{1}{5 \kappa_{5}^{2} a_{1}}\left[\frac{A_{1}}{t^{2}}+c\left(\frac{6 B(2 B-1)}{t^{2}}\right)^{n}\right],
$$




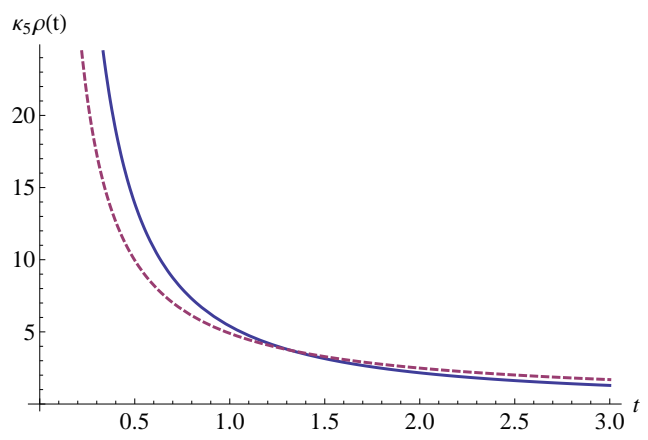

Figure 2: plot of $\rho(t)$ as a function of $t$ for $f(\mathcal{R})=\mathcal{R}+\mathcal{R}^{n}$, dotted curve and for $f(\mathcal{R})=\mathcal{R}^{n}$, solid curve, both for $n=\sqrt{2}$.

where

$$
\begin{aligned}
& A_{1}=A_{2}=\frac{24 n(2 n-1)^{3}(n-1)^{2}(4 n-5)^{2}}{(n-2)^{4}} \\
& c=-\frac{n(n-1)(2 n-1)^{2}(4 n-5)}{(n-2)^{2}} \\
& a_{1}=a_{2}=B(B-1) .
\end{aligned}
$$

In the case $f(\mathcal{R})=\mathcal{R}+\mathcal{R}^{n}$ we have the solution

$$
\omega=-1 \text { for } B \neq 0, \quad B(n)=\frac{7 n-4 \pm \sqrt{n(4 n-1)\left(8 n^{2}-7 n+2\right)}}{8 n^{2}+3 n-8} .
$$

Figure 2 shows a plot of $\rho(t)$ as a function of $t$ for $n=\sqrt{2}$ in both $f(\mathcal{R})=\mathcal{R}^{n}$ and $f(\mathcal{R})=\mathcal{R}+\mathcal{R}^{n}$ cases, taking the plus sign in $B(n)$ above. Using equation (47) we find $B=1.47$ which would point to an accelerated expanding universe.

\section{Conclusions}

In this paper we have studied a brane world scenario in which the bulk action consists of a generic function of the Ricci scalar $f(\mathcal{R})$. The field equations on the brane were then obtained using the SMS method. In this process we obtained the quantity $Q_{\mu \nu}$ which originates from the geometry of the bulk space. For a conformally flat bulk, this quantity is conserved and could therefore be identified with a new kind of matter. We also investigated the static and cosmological solutions of the field equations on the brane and found that the former could be used to explain the galaxy rotation curves while the latter gives the observed behavior for the scale factor at late times.

\section{References}

[1] M. Green, J. Schwarz, and E. Witten, Superstring Theory (Cambridge Monographs on Mathematical Physics) two volumes, Cambridge University Press, 1987; Lust and Theisen, Lectures on String Theory Springer Verlag, 1989; J. Polchinski, String Theory Cambridge Monographs on Mathematical Physics, two volumes, Cambridge University Press, 2004; B. Zweibach, A First Course in String Theory Cambridge University Press, 2004.

[2] L. Randall and R. Sundrum, Phys. Rev. Lett. 83 (1999) 3370 arXiv:hep-ph/9905221

[3] L. Randall and R. Sundrum, Phys. Rev. Lett. 83 (1999) 4690 arXiv:hep-th/9906064

[4] W. Israel, Nouvo Cimento B 44 (1966) 1 . 
[5] T. Shiromizu, K. Maeda, M. Sasaki, Phys. Rev. D 62 (2000) 024012 arXiv:gr-qc/9910076

[6] P. Binetruy, C. Deffayet and D. Langlois, nucl. Phys. B 565 (2000) 269 arXiv:hep-th/9905012; P. Binetruy, C. Deffayet, U. Ellwanger and D. Langlois, Phys. Lett. B 477 (2000) 285 arXiv:hep-th/9910219.

[7] N. Dadhich, R. Maartens, P. Papadopoulos and V. Rezania, Phys. Lett. B 487 (2000) 1 arXiv:hep-th/0003061.

[8] T. Harko and M. K. Mak, Phys. Rev. D 69 (2004) 064020 arXiv:gr-qc/0401049; T. Harko and M. K. Mak, Phys. Rev. D 70 (2004) 024010 arXiv:gr-qc/0404104.

[9] G. Dvali, G. Gabadadze and M. Porrati, Phys. Lett. B 485 (2000) 208 arXiv:hep-th/0005016.

[10] C. Deffayet, Phys. Lett. B 502 (2001) 199 arXiv:hep-th/0010186.

[11] S. Jalalzadeh and H. R. Sepangi, Class. Quant. Gravity 22 (2005) 2035 gr-qc/0408004.

[12] M. Heydari-Fard, H. Razmi and H. R. Sepangi, Phys. Rev. D 76 (2007) 066002 arXiv:0707.3558.

[13] M. Heydari-Fard, M. Shirazi, S. Jalalzade and H. R. Sepangi, Phys. Lett. B 640 (2006) 1 gr-qc/0607067.

[14] K. Maeda, T. Torii, Phys. Rev. D 69 (2004) 024002 hep-th/0309152.

[15] M. Heydari-Fard, H. R. Sepangi, Phys. Rev. D 75 (2007) 064010 arXiv:gr-qc/0702061.

[16] K. Konya, Class. Quant. Grav. 24 (2007) 2761 arXiv:gr-qc/0605119.

[17] K. Atazadeh, M. Farhoudi and H. R. Sepangi, Phys. Lett. B 660 (2008) 275 [arXiv:grqc/0801.1398v1].

[18] V. I. Afonso, D. Bazeia, R. Menezes, A. Yu. Petrov, Phys. Lett. B 658 (2007) 71, [hepth/0710.3790].

[19] J. Saavedra and Y. Vasquez [arXiv: gr-qc/0803.1823v2]; ibid arXiv:hep-th/0601213; ibid [arXiv:astro-ph/0801.4843].

[20] L. P. Eisenhart, Riemannian geometry (Princeton University Press, Princeton, NJ, 1966).

[21] A. Chamblin, S. W. Hawking, H. S. Reall, Phys. Rev. D 61 (2000) 065007. 